\title{
Grundlagen der Grenzen des Strafens
}

\section{Anmerkungen zum Symposium Junger Strafrechtlerinnen und Strafrechtler vom 15. bis 17. Novem- ber 2013 in Frankfurt am Main („Grundlagen und Grenzen des Strafens“)}

Tobias Reinbacher $^{1 *}$

In der letzten Zeit konnte ich mich mehrfach dabei beobachten, wie ich in ziemlich emotional geführten Diskussionen mit fachfremden Gesprächspartnern, welche sich um Strafrecht und die Frage der adäquaten Bestrafung von Straftätern drehten, um Sachlichkeit und um das Aufzeigen der Grenzen bemüht war. Es ging um Pädophilie, um den empfundenen Anstieg der Kriminalität auf den Straßen Berlins, letztlich um die Forderung nach einem härteren Durchgreifen des Staates. Doch nicht nur die Vorlesung Forensische Psychiatrie, die ich vertretungsweise an der LMU München mitveranstalten darf, hat es mir noch einmal deutlich vor Augen geführt: (Vermeintliche) Straftäter sind Menschen mit eigener Geschichte, Kindheit und Sozialisation, sie sind Mitbürgerinnen und Mitbürger, die selbstverständlich auch (Grund-)Rechte haben. In diesen liegen rechtliche Grenzen des Strafens, und dies zu betonen, ist wichtig, gerade auch in den schwierigen Fällen schwerer Straftaten.

Das Strafrecht kann aber auch an faktische Grenzen der Durchsetzbarkeit und der konkreten Strafverfolgung stoßen. Es ist inzwischen ein Allgemeinplatz, dass unsere globalisierte Welt neue Gefahren bereithält, bestimmte Medien forcieren sehr gerne die dahingehenden Ängste, die Straftäter als besondere und qualitativ veränderte Bedrohung erscheinen lassen. Aber ganz nebenbei: Sind die Gefahren heute tatsächlich größer? Man muss gar nicht bis ins Mittelalter zurückblicken, um sich klar zu machen, dass wir heute nicht unbedingt insgesamt gefährlicher leben. Kriminologinnen und Kriminologen mögen das Nähere erläutern und kamen erfreulicherweise auch auf der Tagung des Jungen Strafrechts, der Vereinigung der Jungen Strafrechtlerinnen und Strafrechtler, in Frankfurt am Main zu Wort. So hat namentlich Grischa Merkel uns gezeigt, wie sehr empfundene und reale Bedrohungen auseinander liegen können.

Wir der Rechtswissenschaft Verschriebene haben das Privileg des tieferen Einblicks in die Hintergründe des Strafens, aber auch der fachlichen Distanz. Da ist es unsere Pflicht, auch die Schranken der Reaktionen und Maßnahmen des Staates aufzuzeigen, d.h. nicht nur die Grenzen des Strafens, sondern auch der Überwachung, der Eingriffe in Grundrechte im Dienste der Aufklärung oder gar der Verhinderung von Straftaten, d.h. der Abwehr von (angeblichen) Gefahren. Und wenn schon über Gefahrenabwehr gesprochen wird, so stellen sich weitere Fragen, welche wir Juristinnen und Juristen versuchen sollten, aus ebenjener sachlichen Perspektive zu betrachten: Welche Gefahren rechtfertigen welche staatlichen Maßnahmen? Selbst ein Überwa-

1 * PD Dr. Tobias Reinbacher hat an der Humboldt-Universität zu Berlin habilitiert und vertritt derzeit einen Lehrstuhl an der Ludwig-Maximilians-Universität München. 
chungsstaat könnte uns ja nicht vor allen Gefahren schützen. Wichtiger noch: Wie stark darf in die Rechte der Einzelnen, und zwar sowohl der konkret beschuldigten als auch der nicht beschuldigten Bürgerinnen und Bürger im Allgemeinen, eingegriffen werden? Und soll das Strafrecht überhaupt zur Prävention künftiger Taten dienen? Es geht um nichts Geringeres als die Verteidigung der Freiheit gegenüber staatlichen Eingriffen, deren besonders intensive Ausprägung das Strafrecht ist. Beatrice Brunhöber macht sich schon seit einiger Zeit Gedanken um die Legitimität des Strafrechts, das sie aus der Sicht der Grundrechte als Ergebnis einer Abwägung von Freiheitsrechten versteht, und sie hat sich auch auf dieser Veranstaltung im Eingangsreferat darum verdient gemacht, die diesbezüglichen Grenzen zu ziehen. Aber auch in anderen Vorträgen dieses Symposiums trat immer wieder die Verhältnismäßigkeit als wichtiges Begrenzungsprinzip aus der Sicht der betroffenen Grundrechte in den Vordergrund.

Frankfurt wäre nicht Frankfurt, wenn die Veranstalterinnen und Veranstalter das in diesem Jahr dort abgehaltene Symposium des Jungen Strafrechts nicht zum Anlass genommen hätten, einen kritischen Diskurs um die Schranken des Strafrechts anzustoßen. Ich erinnere mich noch gut an eine meiner ersten Vorlesungen im Ersten Fachsemester Jura, als ein Strafrechtsprofessor dem überraschten Auditorium erläuterte, welche Straftaten seiner Meinung nach abgeschafft werden müssten, weil sie, wie etwa der Ladendiebstahl oder der Eigenkonsum weicher Drogen, mit der ultimaratio-Funktion des Strafrechts schlichtweg nicht vereinbar seien. Ich habe damals gestaunt und erst in späteren Jahren wurde mir klar, wie sehr Frankfurt am Main als Studienort mich geprägt und mir eine kritische Haltung gegenüber der eigenen Disziplin bewahrt hat. Grenzen des Strafrechts können, wie gesagt, faktischer wie rechtlicher Art sein. In ersterer Hinsicht gilt es zu fragen: Was kann das Strafrecht überhaupt leisten? In letzterer hingegen: Was darf es und was muss es leisten? Diesbezüglich lassen sich Grenzen z.B. aus der Sicht der Verfassung ziehen, sei es durch das deutsche Grundgesetz oder sei es durch die europäischen Verträge, Grund- und Menschenrechte. Es kann sich sogar herausstellen, dass wir zu wenig Strafrecht haben, etwa um die Rechtsgüter der EU ausreichend zu schützen, oftmals aber gerade auch, dass das Strafrecht viel zu weit geht und sich von seinen Grundprinzipien entfernt, was uns Frankfurter Kommilitoninnen und Kommilitonen schon in der Erstsemester-Vorlesung aufgezeigt wurde.

An dieser Stelle ist es allerdings an der Zeit, herauszustellen, dass natürlich nicht nur die Goethe-Universität Frankfurt diese Tagung veranstaltet hat. Es war ein Dreigestirn, das hier zusammenwirkte, nämlich die strafrechtlichen Fachbereiche der Juristischen Fakultäten der Universitäten Frankfurt, Hagen und Würzburg. Personell waren Denis Basak, Milan Kuhli, Marc Reiß und Sascha Ziemann für Frankfurt, Martin Asholt für Hagen sowie Susanne Beck und Nina Nestler (noch) für Würzburg 
die Ausrichterinnen und Ausrichter dieses Symposiums. Frankfurt am Main war allerdings der Veranstaltungsort und als solcher nicht nur wegen der beeindruckenden und - zumindest für mich - neuen Räumlichkeiten der Juristischen Fakultät oder der zentralen Lage innerhalb von Deutschland gut gewählt, sondern gerade auch wegen des soeben heraufbeschworenen Frankfurter Geistes.

Frankfurt wäre aber auch nicht Frankfurt, wenn die Frage nach den Grenzen des Strafrechts nicht auch mit derjenigen nach seinen Grundlagen verbunden worden wäre. Es ging den Ausrichterinnen und Ausrichtern darum, die Kritik an den „erheblichen Veränderungen“ im materiellen Recht wie der Rechtsanwendung „neu zu verorten“, um nichts weniger also als um eine „,bewusste Systematisierung“. Ein insgesamt äußerst trefflich gewähltes Thema, das nicht nur zum Ort passt, sondern auch tatsächlich in beeindruckender Vielfalt behandelt wurde. So kamen verfassungsrechtliche wie unionsrechtliche, kriminologische wie demokratietheoretische Überlegungen zur Sprache. Es wurde über künftige Reformen ebenso nachgedacht wie über historische Wurzeln solch reformerischen Gedankengutes. Die Beiträge reichten von der Frage der Prävention durch Strafrecht (Beatrice Brunhöber), über die ReRationalisierung des Strafrechts anhand der Gedanken Franz v. Liszts (Luka Breneselovic), die Grenzen des Geltungsbereichs des Strafrechts (Anne Schneider) sowie der sexuellen Autonomie (Stefan Klingbeil), die Frage der Strafwürdigkeit tatvorbereitender computervermittelter Kommunikation (Stefanie Bock \& Stefan Harrendorf), der Behandlung der Hangtäter (Grischa Merkel) bis hin zu demokratischen Schranken des Strafrechts vermittels der personalen Rechtsgutslehre (Antonio Martins).

Was mich besonders begeistert, ist aber eine Form der Durchbrechung von Grenzen, welcher die Symposien des Jungen Strafrechts ganz generell verschrieben sind. Sie sind nämlich nicht nur Treffen der Habilitandinnen und Habilitanden im Strafrecht, sondern öffnen sich auch für Doktorandinnen und Doktoranden, Wissenschaftliche Mitarbeiterinnen und Mitarbeiter sowie für Studierende. Sie sind gedacht als Forum, um „dem wissenschaftlichen Nachwuchs im deutschsprachigen Raum die Möglichkeit zu eröffnen, sich regelmäßig fachlich miteinander auszutauschen und zu vernetzen" (diese Hinweise finden sich im Tagungsprogramm). Sie sind folglich insofern „grenzenlos“ im Hinblick auf die bereits absolvierten Ausbildungsschritte. Was in dieser Hinsicht schon in Köln und Berlin begonnen wurde, konnte hier sehr erfolgreich fortgesetzt werden. Letztlich hatten denn auch zwei der sieben Referentinnen und Referenten ihre Promotion zur Zeit ihres Vortrages noch nicht abgeschlossen. Gerade sie sind an der hier betriebenen Grundlagenforschung beteiligt und haben selbstverständlich ihre eigene Position zu den Grenzen des Strafrechts, sodass ihre Teilnahme am Symposium nicht nur gerechtfertigt, sondern meines Erachtens durchaus notwendig war und ist. 
Es ist ein Kennzeichen des Jungen Strafrechts, dass die Veranstaltung insofern keine Grenzen zieht und sich nicht als elitärer Zirkel verstehen will. Die Symposien sind einem offenen Diskurs verschrieben, der ohne große formale Zwänge abläuft und auch deshalb stets bereichernd ist. Wir haben auf dem Frankfurter Symposium viel lernen können über die Grundlagen und Grenzen des Strafrechts. Genauer gesagt hat das Symposium die Grundlagen der Grenzen aufgezeigt, wenn man so sagen darf. Denn die Beiträge gingen an die Substanz dessen, was wir heute wohl ein „modernes Strafrecht" nennen.

Beatrice Brunhöber (Humboldt-Universität zu Berlin) behandelte unter der Überschrift „Prävention durch Strafrecht?“ im Eingangsreferat die Frage der Vermischung von Strafrecht und Polizeirecht. Wenn der Staat eingreift, bevor es überhaupt zu einer Straftat gekommen ist, so darf durchaus festgestellt werden, dass er hier eher Gefahrenabwehr denn Sanktion im Sinne hat. Die Referentin wies darauf hin, dass die entsprechenden Gesetze schon im Titel deutlich machen, worum es ihnen geht, nämlich um „Bekämpfung“. Ein Phänomen, das sie für Deutschland behandelte, welches aber, so darf ergänzt werden, auch aus dem EU-Recht nicht unbekannt ist. Es ging ihr dabei um Erklärungen dieser Entwicklung, letztlich aber, ganz am Oberthema orientiert, selbstverständlich auch um Antworten, also gerade darum, welche Grenzen bei diesem präventiv ausgerichteten Strafrecht zu ziehen sind. Da die Referentin (eher) präventiv ausgerichtete Strafnormen als Faktum anerkannte, plädierte sie für eine Akzentverschiebung: Es gehe diesen Strafnormen nicht, wie häufig angenommen, um die Abwehr von Gefahren, sondern um eine Steuerung von Risiken. Sinnt man über mögliche Grenzen solcher Strafvorschriften nach, und legt man dabei diese vorgeschlagene Ausrichtung an der Steuerung von Risiken zu Grunde, so bietet es sich an, kriminologisch und empirisch zu fragen, ob die entsprechenden Risiken überhaupt bestehen. Wenn nicht, dürfte es an der Legitimität der Strafvorschriften mangeln.

Bzgl. der notwendigen normativen Schranken blieb Beatrice Brunhöber ihrer Linie treu und orientierte sich an den Vorgaben der Grundrechte. Verbote und darauf bezogene strafrechtliche Sanktionen müssen als Grundrechtseingriffe verhältnismäBig sein. Das Ziel müsse es dann sein, für präventive, d.h. in ihrer Lesart: an Risiken orientierte, Strafnormen spezifische Verhältnismäßigkeitskriterien zu entwickeln. Denn es sei zu berücksichtigen, dass Freiheitseingriffe durch Verbote, die „nur“ Risiken steuern sollen, einer besonderen Rechtfertigung bedürftig seien. Im weiteren Verlauf der Tagung wurde klar, dass das Eingangsreferat hier eine Grundlage für die ganze Veranstaltung gelegt hat, auf die sich im Zuge der anderen Beiträge bestens zurückkommen ließe, nämlich die Begrenzung des Grundrechte beschränkenden Strafrechts durch das Verhältnismäßigkeitsprinzip. 
Thematisch (nur) augenscheinlich anders ausgerichtet war der Vortrag von Luka Breneselovic (Ludwig-Maximilians-Universität München) „Kann und soll die bevorstehende Re-Rationalisierung des Strafrechts auf den Gedanken und der Taktik von Franz v. Liszt aufbauen?" Ihm ging es, salopp gesprochen, um eine Art Rehabilitierung Franz v. Liszts, dessen Schriften er teilweise für zu kritisch rezipiert erachtet. Letztlich habe ich aber auch seinen Ausführungen Hinweise auf eine Notwendigkeit kriminologischer Rückbindung strafrechtlicher Normen entnommen. Eine Re-Rationalisierung des Strafrechts, die - nicht nur in den oben angeführten Gesprächen, sondern erst recht auch in der Strafrechtspolitik - dringend notwendig erscheint, lasse sich, so der Referent, auch mit den Lehren v. Liszts fundieren. Denn schon dieser habe sich die Praxis, das tägliche Leben, angeschaut und sodann festgestellt, in welchen Bereichen zu viel Strafrecht operiert. Gerecht sei für ihn nur das gewesen, was notwendig ist. Hier liegt eine sinnvolle Begrenzung des Strafrechts durch praktisches Denken, die keinerlei Aktualität verloren hat.

Die europäische Dimension addierte der Vortrag von Anne Schneider (Rheinische Friedrich-Wilhelms-Universität Bonn) „Grenzen des Geltungsbereichs von Strafrecht", der sich mit dem Strafanwendungsrecht beschäftigte, d.h. mit der Frage einer Beschränkung der Strafgewalt bei transnationalen Sachverhalten. Solche Schranken überprüfte die Referentin aus der Sicht des Völkerrechts, des EU-Rechts sowie des nationalen Verfassungsrechts. Während das Völkerrecht den „souveränen“ Nationalstaaten noch viel Freiheit zur autonomen Bestimmung der Reichweite ihrer Strafgewalt lässt, stellt sich dies - wie in anderen Bereichen des materiellen und prozessualen Rechts auch - auf der Ebene der EU ganz anders dar. Hier haben sich insbesondere die Grundfreiheiten in der Vergangenheit - bei grenzüberschreitenden Sachverhalten - schon häufig als besondere Grenze nationaler Strafnormen erwiesen. Daher ist es nur konsequent bzw. meines Erachtens sogar unumgänglich, auch bei den $\mathbb{S} 3 \mathrm{ff}$. StGB zu überprüfen, ob nicht etwa eine unzulässige Beschränkung der durch das EU-Recht gewährten Rechte vorliegt. Innerhalb der EU sind die Mitgliedstaaten keineswegs mehr völlig frei, die Grenzen ihres Strafrechts selbst zu bestimmen, sondern müssen das EU-Recht, das mit einem Anwendungsvorrang ausgestattet ist, beachten. Nationale Regeln müssen sich diesem im Falle eines Konflikts beugen, d.h. sie dürfen für Sachverhalte, in denen sie mit den entsprechenden EU-Bestimmungen kollidieren, nicht mehr angewendet werden.

In dieser Logik ist es nur auf den ersten Blick überraschend, dass die Referentin sowohl das passive als auch das aktive Personalitätsprinzip des $\$ 7$ StGB im Hinblick auf das Diskriminierungsverbot des Art. 18 AEUV als unionsrechtswidrig verabschieden will. Im Hinblick auf Ersteres lässt sich doch durchaus fragen, ob in einer Union mit gemeinsamem Indigenat die nationale Staatsangehörigkeit tatsächlich noch das geeignete Kriterium ist oder ob die Jurisdiktion sich nicht vielmehr am 
Wohnort orientieren müsste. Beim aktiven Personalitätsprinzip musste man aber schon genauer hinhören, sind doch Inländerdiskriminierungen unionsrechtlich zunächst einmal nicht verboten. Hier stellte die Referentin darauf ab, dass bei Auslandstaten eben kein rein inländischer Sachverhalt mehr vorliege, die Täter vielmehr von ihrem Freizügigkeitsrecht Gebrauch gemacht hätten und daher auch nach ihrer Rückkehr in den eigenen Staat nicht sanktioniert, also schlechter als andere Unionsbürger gestellt, werden dürften, sonst würden sie wohl von ihrer Freizügigkeit keinen Gebrauch machen. Hier übertrug sie Grundsätze, die der EuGH in anderen Konstellationen entwickelt hat. Falls dies (noch) nicht überzeugt, fand Anne Schneider noch einen zweiten Begründungsstrang: Da es für den Täter häufig vorteilhaft sei, wenn das Verfahren vor den Gerichten seines Heimatstaates geführt werde, liege hierin eine Diskriminierung anderer Unionsbürgerinnen und Unionsbürger. Denn immerhin könnten ja selbst solche EU-Bürgerinnen und EU-Bürger, die schon seit vielen Jahren in Deutschland leben, hier also ihren Lebensmittelpunkt gewählt haben, sich bei Auslandtaten nicht auf das aktive Personalitätsprinzip berufen, um in Deutschland ein Verfahren zu erhalten. Mir sind solche Überlegungen durchaus sympathisch. Die EU bildet zwar keinen Bundesstaat, letztlich legen die Unionsbürgerschaft und die offenen Grenzen es meines Erachtens aber nahe, sich von am Gedanken der Staatsangehörigkeit geprägten Strafanwendungsregeln zu verabschieden. Beim letzten Punkt, der Überprüfung des Strafanwendungsrechts anhand der deutschen Verfassung, schloss sich schließlich wiederum der Kreis zum Vortrag von Beatrice Brunhöber, denn auch Anne Schneider erkannte das Verhältnismäßigkeitsprinzip als verfassungsmäßige Schranke an, und in Anwendung desselben verwarf sie \$ 7 II Nr. 2 StGB letztlich als unverhältnismäßig.

„Strafrecht und Moral“ - dies wäre eine weiteres großes Thema, das nach einer umfassenden Bearbeitung im Rahmen eines Symposiums verlangen könnte. Einen Teilausschnitt behandelte Stefan Klingbeil (Humboldt-Universität zu Berlin) unter der Überschrift „Grenzen sexueller Autonomie in der konstruierten Wirklichkeit“ jedoch schon in Frankfurt. Den Fixpunkt seiner Analyse bildete der sexuelle Missbrauch von Schutzbefohlenen gemäß $\$ 174$ StGB, wobei der Fokus auf dem Verhältnis von Lehrkräften und Schülerinnen oder Schülern lag. Hier erachtete der Vortragende die durch die Rechtsprechung gezogenen Grenzen des Strafens, genauer: ihre hierzu herangezogenen Kriterien, für wenig überzeugend. Diese stellt nämlich auf den Einzelfall ab, um zu beurteilen, ob es sich um ein Anvertrautsein zur Erziehung handelt. Anders als der Klassenlehrer, hat ein Lehrer, der die Schüler sonst nicht unterrichtet, etwa (nur) während der Pausenaufsicht die nach \174 Abs. 1 Nr. 1 StGB erforderliche Stellung, sodass nach Ertönen des Pausengongs ein solches Verhältnis enden soll, ein sexuelles daher beginnen darf. In ähnlicher Weise soll es etwa bei Vertretungsstunden auf deren Anzahl ankommen. Auf diese Weise entstehen relative oder nur temporäre Obhutsverhältnisse. Den Referenten 
überzeugt dies nicht, da hier eine Einzelbetrachtung anhand diffuser Kriterien angestellt werde, anstatt generell anzuerkennen, dass typischerweise ein institutionalisiertes Subordinationsverhältnis zwischen den Schülerinnen und Schülern und den Lehrerkräften bestehe. Das Rechtssystem solle sich hier lieber in Zurückhaltung üben, d.h. seine eigenen Grenzen (der Beurteilung!) erkennen, und auf diese Fallgestaltungen mit einer klaren Trennlinie reagieren.

Da das Gesetz zudem nicht auf den natürlichen Willen der Betroffenen abstelle, sondern vielmehr normativ festlege, dass unter 18 Jahren die sexuelle Selbstbestimmung noch äußerst fragil sei, erschien ihm unter diesem Gesichtspunkt auch die Einschränkung der Strafbarkeit des Abs. 4 eher inkonsequent. Denn das Gesetz will das Verhalten der Schülerinnen und Schüler nun doch berücksichtigen. So kann etwa „echte Liebe“ zwischen den Beteiligten hiernach dem Gericht die Möglichkeit zum Absehen von Strafe für den Lehrer eröffnen. Wo also liegen hier die Grenzen der Strafgerichte? Doch wohl darin, dass es schwer sein dürfte, die Frage nach der echten Liebe abschließend zu beurteilen. Haben wir hier also ein Beispiel für zu wenig Strafrecht gefunden, d.h. für Grenzen des Strafens, die es zu beseitigen gilt? Die Odenwaldschule liegt jedenfalls nicht weit entfernt von Frankfurt am Main. Stefan Klingbeil ging es in seinem Vortrag jedoch nicht in erster Linie darum, die rechtspolitische Frage nach der Strafwürdigkeit dieser Konstellationen, und damit der Grenzen des Strafens in diesem Bereich, abschließend zu beantworten. Vielmehr war es sein Ziel, die psychologische Komplexität dieser Fallgestaltungen herauszuarbeiten und aufzuzeigen, dass das Strafrecht gut daran tun würde, mit klaren Kriterien zu antworten.

Grundlagenforschung anderer Art betrieben Stefanie Bock und Stefan Harrendorf (Georg-August-Universität Göttingen). Sie untersuchten „Strafbarkeit und Strafwürdigkeit tatvorbereitender computervermittelter Kommunikation“. Bei dieser Thematik kommen beide oben angesprochenen Grenzen des Strafrechts/Strafens zum Tragen. In faktischer Hinsicht muss man die gerade durch die Anonymität im Internet bedingten Schwierigkeiten der Ermittlung der Täter in Betracht nehmen. In rechtlicher Hinsicht ist genau zu prüfen, wann Äußerungen im Internet einen Straftatbestand erfüllen. Dabei geht es nicht nur um die Anwendbarkeit des deutschen Strafrechts auf Internet-Sachverhalte, sondern auch um die ganz konkrete Subsumtion unter vorhandene Straftatbestände. Dies gilt umso mehr deshalb, weil die beiden Referenten gerade das Stadium der Tatvorbereitung untersuchten. Hier wirkt sich die Anonymität des Internets in einer Weise aus, die es erfordert, genau hinzusehen. Denn ist es nicht deutlich leichter, etwas „daher zu sagen“, wenn man sich gänzlich unerkannt wähnt? Das muss nicht unbedingt gegen eine Strafbarkeit sprechen, aber doch auch nicht dafür. So hat der BGH im Rahmen der Prüfung einer anonymen Verbrechensverabredung in einem Internet-Chat zu Recht eine Strafbarkeit nach $\$ 30$ Abs. 2 StGB abgelehnt, da keine Möglichkeit bestand, den Chatpartner zu iden- 
tifizieren. Die erforderliche Willensbindung ließ sich dort nämlich nicht nachweisen. Und schließlich stellt sich die Frage nach der Strafwürdigkeit entsprechender Verhaltensweisen in ganz besonderem Maße im Vorbereitungsstadium. Wiederum lässt sich der Bogen spannen zu Beatrice Brunhöbers Eingangsreferat: Bei Sanktionen im Stadium der Tatvorbereitung handelt es sich eigentlich um Prävention (bzw. um Risikosteuerung), sodass eine „Vermischung mit dem Polizeirecht“ zu befürchten und eine besondere Verhältnismäßigkeitsprüfung anzustellen ist.

Stefanie Bock und Stefan Harrendorf haben daher ganz zu Recht in ihrem Referat nicht nur die Strafbarkeit de lege lata (Strafbare Kommunikation, Vorfeld von Sexual- und Gewaltstraftaten und Vorstufen der Beteiligung nach $\mathbb{3} 30 \mathrm{StGB}$ ) aufgezeigt, sondern gerade auch die Frage nach der Strafwürdigkeit solcher Verhaltensweisen aufgeworfen. Dabei ging es (auch) ihnen um ein strafbarkeitsbegrenzendes „kritisches Konzept zur Feststellung des Bedarfs an Kriminalisierung und Entkriminalisierung“. Hier haben sie, durchaus im Einklang mit den vorangegangenen Referaten, ebenfalls das Verhältnismäßigkeitsprinzip zur Anwendung gebracht, es allerdings nicht dabei belassen. Vielmehr solle die Frage der Strafwürdigkeit eines Verhaltens auch anhand originär strafrechtlicher Grenzen beurteilt werden. So müssten auch ein sozial-ethischer Vorwurf gerechtfertigt und eine Kohärenz mit anderen Strafnormen hergestellt werden. Welche Vorbereitungshandlungen rechtfertigen aber einen sozial-ethischen Vorwurf? Dies ist ein großes Thema, weshalb auch die Referenten darauf hinwiesen, dass sich hieraus durchaus ein umfassendes Forschungsprojekt entwickeln ließe, selbst wenn man es auf die computervermittelte Kommunikation konzentrieren wollte. Hier könnte also dieser Vortrag eine Grundlage liefern, und damit letztlich wiederum eine Grundlage der Grenzen einer solchen Vorfeldkriminalisierung. Wichtig erscheint mir, dass die beiden Vortragenden ihre Beurteilung der Strafwürdigkeit neben dem Wert des Rechtgutes und der Verwerflichkeit der Tätergesinnung gerade auch an der Gefährlichkeit des Rechtsgutangriffs festmachen wollten, denn dabei können (auch) sie wiederum kriminologische Erkenntnisse fruchtbar machen.

Haben wir es mit realen Bedrohungslagen zu tun oder empfinden wir diese nur, z.B. wegen eines besonderen medialen Interesses? Diese Frage habe ich einleitend schon aufgeworfen. Wenn wir Juristinnen und Juristen nun realistisch, Luka Breneselovic könnte sagen: aus einer „re-rationalisierten“ Perspektive, die Gefahrenlage einschätzen wollen, so brauchen wir Fakten, welche die Grundlage der Beurteilung bilden. Auch Beatrice Brunhöber hat zu Recht herausgestellt, dass die Risiken erst einmal identifiziert werden müssen, bevor sie mit Strafnormen „bekämpft“ werden können bzw. dürfen. Solche Daten kann uns die Kriminologie liefern. Dies führt uns zum nächsten Referat. 
Es ist erfreulich, dass Grischa Merkel in ihrem Vortrag „Hangtäter, quo vadis?“ im Hinblick auf die in der Sicherungsverwahrung untergebrachten Straftäter eine Vielzahl solcher Hintergrundinformationen und damit wiederum auch Grundlagen der Grenzen des Strafrechts geliefert hat, genauer: des Maßregelvollzuges. Ein interessanter historischer Vergleich zwischen den in der Zeit des Nationalsozialismus untergebrachten „Hangtätern“ mit der heutigen Situation zeigte zwar einerseits den wenig überraschenden exorbitanten zahlenmäßigen Unterschied und die Divergenz der Qualität der Taten (primär Vermögensdelikte damals, primär Sexualdelikte heute), sodass die „Hangtäter“ nur im Hinblick auf die wiederholte Tatbegehung wirklich vergleichbar erscheinen. Er förderte andererseits aber auch erstaunliche Parallelen zu Tage, sowohl soweit das mediale Interesse an den entsprechenden Taten und Tätern betrachtet wurde als auch soweit das (auch daraus resultierende oder immerhin dadurch verstärkte) Bedrohungsgefühl in der Bevölkerung aufgezeigt wurde. Gerade in Bezug etwa auf Sexualmorde an Kindern gehen gefühlte und reale Bedrohungslagen seit Jahren auseinander. Auch der Effekt auf die Einstellung der Gesellschaft gegenüber diesen Täter ist ähnlich: Eine Solidarisierung seitens der Bevölkerung dürfen sie nicht erwarten. Dies haben auch meine oben erwähnten Gespräche belegt. Weiterhin zeigte Grischa Merkel anhand von Studien die Zusammenhänge zwischen bestimmten hirnorganischen Ursachen und der späteren Unterbringung in der Sicherungsverwahrung als Folge wiederholter Straftaten auf. 2/3 der Untergebrachten erfüllten die Merkmale der Psychopathie und der dissozialen Störung, viele stammten aus schwierigen Verhältnissen und seien schon seit der frühen Jugend straffällig, sodass die Frage der Unterbringung nach $\$ 63$ StGB oder nach $\ 66$ StGB vom Zufall abhänge, man möchte ergänzen: oder jedenfalls vom jeweiligen Gutachter. Auch hier konnte ich als Zuhörer eine Bestätigung für das finden, was ich, wiederum auf Grund meiner Erfahrungen aus der Vorlesung Forensische Psychiatrie, schon vermutet hatte.

Besonders wichtig für die Frage möglicher Grenzen der Maßregeln, genauer: Alternativen zu denselben, erschien mir aber die darauf folgende Präsentation von Studien zu den Zusammenhängen von hirnorganischen Störungen und deviantem Verhalten bzw. zum Serotoninhaushalt, der sich hinsichtlich der Entwicklung zu einem Straftäter auswirken kann, aber nicht muss. Hier spielen die Familienverhältnisse eine entscheidende Rolle, etwa Misshandlungen in der Kindheit. Nun einmal angenommen, bestimmte Risikofaktoren sind tatsächlich identifizierbar, was soll unsere Gesellschaft dann tun? Abwarten, bis es zu spät ist, oder vielleicht doch eine Solidarisierung wagen mit Menschen, deren Ausgangslage nun einmal deutlich schlechter ist? Dass Grischa Merkel die zweite Lösung bevorzugt, beinhaltet nicht unbedingt nur ein Eintreten für die eingangs beschriebene fachliche Distanz als Juristin sondern gewissermaßen gleichzeitig auch für das Gegenteil, nämlich für ein Aufgeben von ablehnender sozialer Distanz. Für sie liegt der bessere Weg in der Sozialisierung, nicht 
erst in der Re-Sozialisierung. Ich hätte ihre Unterstützung bei meinen eingangs erwähnten Gesprächen sehr gut gebrauchen können.

Nach allem was wir gehört haben, war es ein gelungener Schachzug der Veranstalterinnen und Veranstalter, an das Ende des Symposiums ein Referat zu setzen, das die nun zwingend logische Frage behandelte, wie der demokratisch legitimierte Gesetzgeber am besten vorzugehen hat, einmal vorausgesetzt, er handelt mit rationalem Vorbedacht. Oder lassen Sie mich anders fragen: Steht es in der Demokratie dem Gesetzgeber völlig frei, ob und welche Strafnormen er schaffen will? Ist es Konsequenz des demokratischen Systems, dass das vom Volk gewählte Parlament, ganz im Sinne der Volkssouveränität, tun und lassen kann, was es will? Die Frage ist nahezu rhetorisch, wurde doch soeben ausgeführt, dass die Verfassung, d.h. insbesondere die deutschen Grundrechte und gegebenenfalls auch das EU-Recht, Grenzen ziehen. Akzeptiert man ferner, dass das Strafrecht nur ultima ratio sein darf und als Grundrechtseingriff verhältnismäßig sein muss, so wird klar, dass hier Schranken der Gesetzgebung liegen. Demokratie und Rechtsstaatsprinzip gehören also zusammen.

Antonio Martins hat sich indessen noch einmal der (personalen) Rechtsgutslehre zugewandt, welche dem Gesetzgeber eine weitere Schranke auferlegen möchte: Strafnormen sind nur legitim, wenn sie auch ein legitimes Rechtsgut schützen. Muss der Gesetzgeber diesen Nachweis erbringen, so darf er beispielsweise Strafrecht und Moral nicht in einer Weise verbinden, dass nur noch eine bestimmte moralisch-ethische Idealvorstellung, nicht aber ein legitimes Rechtsgut, strafrechtlich geschützt wird. Von den Vorwürfen, denen diese Lehre sich ausgesetzt sieht, griff der Referent denjenigen des Konfliktes mit dem Demokratieprinzip heraus. Seine These war es nun, dass die Forderung nach einem legitimen Schutzgut, er möchte es lieber „menschliches Interesse“ nennen, der Gesetzgebung gerade nicht undemokratische Schranken auferlegen muss, sondern vielmehr den demokratischen Rechtssetzungsprozess begleiten kann und muss. Wenn Demokratie und Rechtsstaatsprinzip tatsächlich so eng beieinander liegen, so bedingen sie sich wechselseitig. Das bedeute dann aber auch, dass der Gesetzgeber schon bei seiner demokratischen Entscheidungsfindung die Grenzen beachten muss, die ihm die Rechtsgutslehre zieht. Damit werde deren (berechtigte) Skepsis zu einem (essentiellen) Teil des demokratischen Diskurses. Da es ihm um eine „personale Rechtsgutslehre“ geht, steht für ihn das Individuum im Vordergrund. Ist das demokratische Kollektiv eine Ansammlung von Individuen, so können diese für den Schutz ihrer Interessen sorgen, welche selbstverständlich miteinander in Ausgleich zu bringen sind - kurz gesagt: Interessen finden ihre Grenze in den Interessen des anderen. Antonio Martins versteht Demokratie damit nicht als formalisierte Majoritätsentscheidung, sondern traut ihr zu, bereits innerhalb des Diskurses über die Notwendigkeit von Strafnormen die Schranken zu 
bedenken, welche die Rechtsgutslehre setzt. Diese soll die Demokratie nicht behindern, sondern rechtsstaatlicher machen.

Ein vernünftiger Gesetzgeber wird sich Gedanken machen, ob seine Strafgesetze legitime Ziele verfolgen, ob sie im Einklang mit den Grundrechten und den Vorgaben der EU stehen, und er wird diese Vorgaben insbesondere dann beachten, wenn er Risiken steuern und Vorfeldhandlungen adäquat behandeln will. Er wird hoffentlich auch die Abweichler von der Norm nicht nur als Täter oder gar „Hangtäter“ begreifen, sondern Grenzen beachten, die ihm die Vernunft, aber auch die schlichte Menschlichkeit, diktieren. Tut er dies nicht, so habe ich keine Zweifel, dass die Referentinnen und Referenten des Frankfurter Symposiums ihn gerne an die Grundlagen und Grenzen des Strafens erinnern werden. 